\title{
New Team at the Journal and Birth of the MicroG Network
}

\section{Novo time da Revista e o nascimento da Rede MicroG}

\author{
Thais Russomano1, Rafael Reimann Baptista ${ }^{1}$, Isabel Rocha², Mafalda Carvalho² \\ PUCRS, Microgravity Centre, Brazil. \\ 2 ULISBOA, Microgravity Centre, Portugal.
}

- dition number 1, volume 5 of Aviation in Focus - Journal of Aeronautical Sciences, brings Etogether three original articles, one review and an interview. This edition also marks a change in the Editorial Board of the publication, with Prof. Thais Russomano, (Microgravity Centre/School of Medicine, PUCRS) assuming the position of Editor and Prof. Rafael Reimann Baptista (Microgravity Centre/Faculty of Physical Education, PUCRS) acting as Executive Editor.

The first article in this issue, entitled Analysis of financial impact resulting from option for aircraft noise reduction against operational optimization at Brasilia International Airport, performs an analysis of the financial impact on the air transport system from the adoption of longer aircraft taxiing routes, due to noise pollution.

The second article, ANACpedia: aviation, presents the ANACpedia, a database made up of aviation industry vocabulary in the form of lists of terms, composed of two bilingual glossaries (English-Portuguese/Portuguese-English, Spanish-Portuguese/Portuguese-Spanish) and a list English acronyms.

The third article, entitled Women in aviation: less posing, more action, aims to investigate the profile and media representation of female professionals in aviation.

The fourth article, Leadership approaches in multi-cultural aviation environments, gives a detailed analysis through a literature review of leadership approaches in multi-cultural working environments within the aviation industry.

The edition closes with an interview with researcher Agustin Folgueira, Professor of Neuroanatomy at the University of Buenos Aires. He is currently working as a doctor at the Belgrano II Base in Antarctica, in addition to developing a study related to the Mars 500 project.

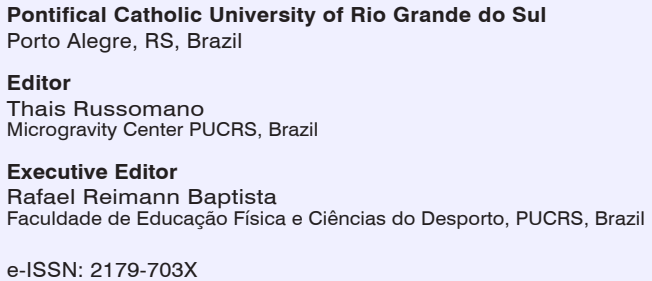

Corresponding Author:

Thais Russomano

trussomano@hotmail.com

Received: November 02, 2014 Accepted: November 02, 2014

(C) 2014 EDIPUCRS 
Agustín participated in the online distance learning course in Space Life Sciences, promoted by the Microgravity Centre, PUCRS, and tells us about his experience in Antarctica.

In addition to the announcement regarding Editorial Board changes of Aviation in Focus, another piece of good news is the creation of the MicroG-Lisbon in October, 2014. Although physically located in the Institute of Physiology at the Faculty of Medicine, Lisbon, the MicroG-Lisbon counts not only on members of the Faculty of Medicine, but also on elements of the Portuguese Air Force.

The group, led by Prof. Isabel Rocha and assisted by Mafalda Carvalho, MSc, aims to develop aviation and space sciences at the university of Lisbon in particular, and also within the MicroG Labs international network. To this end, a variety of academic and research activities will be carried out in these scientific areas with other network partners, but especially in this early phase, with the MicroG-PUCRS.

In this way, it is hoped to contribute to the exploration and achievement of a high level of collaborative opportunities, benefitting from the multidisciplinarity of the teams involved. It should also promote the development of an international-scale culture that combines research, education and innovation under the MicroG Labs Network vision. 